\title{
DESENVOLVIMENTO DE UMA FERRAMENTA COMPUTACIONAL SEGUNDO O MÉTODO DOS ELEMENTOS FINITOS PARA A ANÁLISE DE VIGAS DE CONCRETO ARMADO REFORÇADAS COM FIBRAS NATURAIS
}

\author{
Paulo Henrique Silva dos Santos ${ }^{1}$; Paulo Roberto Lopes Lima²; \\ 1. Bolsista PIBIC/CNPq, Graduando em Engenharia Civil, Universidade Estadual de Feira de Santana, e-mail: \\ pauloenghenrique@gmail.com \\ 2. Orientador, Departamento de Tecnologia, Universidade Estadual de Feira de Santana, e-mail: \\ lima.prl.uefs@gmail.com
}

\section{PALAVRAS-CHAVE: vigas de concreto; fibras vegetais; elementos finitos. INTRODUÇÃO}

O avanço no desenvolvimento dos compósitos tem permitido, mesmo com fibras curtas, a produção de materiais cimentícios com comportamento pós-fissuração caracterizado como plástico ou mesmo com aumento da capacidade portante ("strain hardening") [1]. Devido à presença das fibras, o material apresenta várias fissuras, deixando de ser um contínuo, mas mantém a sua integridade a grandes deformações. Com isso, impõe-se a busca de novas soluções teóricas, baseadas, por exemplo, em mecânica da fratura [2], ou mecânica do dano [3] para modelagem dos mesmos sob efeito de cargas externas. Nesse sentido, foi desenvolvido aqui um modelo teórico-computacional para prever o comportamento de vigas de concreto armado reforçadas com capas de matriz cimentícia reforçadas com fibras de sisal, combinando a análise não linear física, através do uso de diagramas tensão-deformação linearizados para os materiais com a Teoria Clássica de Laminados. Foram estudadas somente o caso de capas de matriz cimentícia na forma de perfil $\mathrm{U}$, funcionando inicialmente como forma perdida para a concretagem das vigas de concreto armado. A estratégia de laminar o elemento estrutural (viga de concreto armado reforçada com capa de concreto com fibras) traz a vantagem de permitir a simulação do processo de fissuração progressiva ao longo do elemento.

\section{METODOLOGIA}

Para a construção da ferramenta computacional mencionada acima proposta foi utilizado um programa em Fortran, baseado em Elementos Finitos, desenvolvido e validado anteriormente [4], para a análise de vigas de concreto armado considerando a Teoria Clássica de Laminados e o modelo de dano escalar de Mazars. Por conta da complexidade da tarefa em si primeiramente foi desenvolvida a formulação analítica para esse modelo de vigas e vários casos de vigas de concreto armado, com e sem capa de reforço, foram testados no âmbito mais restrito, qual seja, linear físico (comportamento linear elástico). Tais testes serviram de referência posterior para validação da parte linear física do novo código computacional aqui desenvolvido. Terminada a fase linear do trabalho foram implementadas todas as partes associadas à análise não linear física (relações constitutivas dadas por curvas multilineares), as quais não foram ainda completamente validadas.

\section{FORMULAÇÃ̃ DO PROBLEMA}

No modelo de dano o comportamento tensão-deformação dos materiais é progressivamente danificado de acordo com a equação 


$$
E=(1-D) E_{o}
$$

sendo $E_{o}$ o módulo de elasticidade do material íntegro e $D$ a variável de dano, a qual varia de 0 a 1 . No presente trabalho foram consideradas vigas prismáticas formadas por lâminas de espessura uniforme, sendo adotadas as hipóteses da Teoria Clássica de Laminados, a partir da qual, com base na Lei de Hooke Generalizada, podem ser estabelecidas as seguintes expressões para o trabalho virtual realizado pelas forças internas e externas, respectivamente:

$$
\begin{gathered}
\delta W_{\mathrm{int}}=\int_{0}^{L}\left[\left(A_{11} \frac{\partial u_{0}}{\partial x}-B_{11} \frac{\partial^{2} w_{0}}{\partial x^{2}}\right) \delta\left(\frac{\partial u_{0}}{\partial x}\right)-\left(B_{11} \frac{\partial u_{0}}{\partial x}-D_{11} \frac{\partial^{2} w_{0}}{\partial x^{2}}\right) \delta\left(\frac{\partial^{2} w_{0}}{\partial x^{2}}\right)\right] d x \\
\delta W_{e x t}=\int_{0}^{L}\left[p(x) \delta u_{0}+q(x) \delta w_{0}\right] d x+\left[\bar{F}_{x} \delta u_{0}+\bar{F}_{z} \delta w_{0}-\bar{M} \delta\left(\frac{\partial w_{0}}{\partial x}\right)\right]_{0}^{L}
\end{gathered}
$$

sendo $u_{0}(x)$ e $w_{0}(x)$ os deslocamentos segundo os eixos $x$ e $z$, nessa ordem, do ponto localizado sobre o eixo da viga. As rigidezes $A_{11}, B_{11}$ e $D_{11}$ estão associadas, respectivamente, a matrizes de rigidez extensional $[A]$, rigidez flexional $[B]$ e de acoplamento $[D]$, da teoria de laminados [11]. As grandezas $p(x)$ e $q(x)$ representam as cargas distribuídas de domínio, segundo as direções $x$ e $z$, respectivamente, e nas extremidades $x=0$ e $x=L$ da viga, estão aplicadas as cargas $\bar{F}_{x}, \bar{F}_{z}$ e $\bar{M}$ (essas cargas foram desenhadas com o sentido positivo na Figura 1).

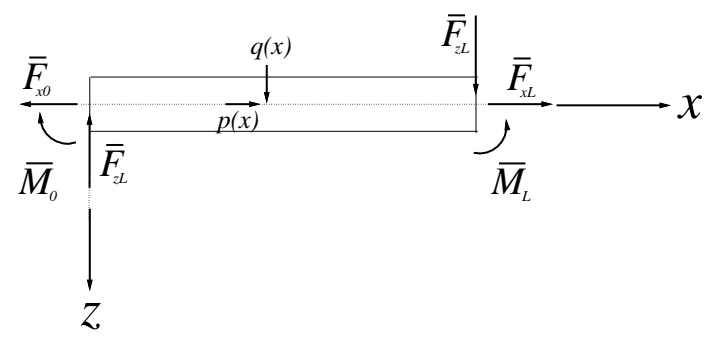

Figura 1. Carregamento considerado na viga

Para o tratamento segundo o Método dos Elementos Finitos (MEF) da formulação, foi escolhido o elemento clássico de viga [14], delimitado por dois nós em suas extremidades, com três graus de liberdade em cada um desses nós, quais sejam: $u_{0}, w_{0}$ e $\frac{\partial w_{0}}{\partial x}$. As funções de interpolação usadas para representar os deslocamentos ao longo do elemento finito de comprimento $L_{e l}$, foram polinomial cúbica para $w_{0}=w_{0}(x) \mathrm{e}$ linear para $u_{0}=u_{0}(x)$. De modo sucinto a aplicação do MEF gera um sistema de equações nodais de equilíbrio do tipo:

$$
[K]\{D\}=\{F\}
$$

onde $[K]$ é a matriz de rigidez da estrutura, função tanto da geometria da viga quanto das propriedades mecânicas dos materiais que a constituem, $\{F\}$ é o vetor de cargas, contendo as ações nodais do carregamento atuante na estrutura, e $\{D\}$ é o vetor de deslocamentos nodais, obtido através da resolução do sistema de equações dado por (23).

\section{RESULTADOS E DISCUSSÃO}


Ao todo, foram testados 5 (cinco) situações de viga: Viga de concreto armado sem a forma perdida (CON), Formas perdida não concretadas com espessuras de 15 e 20 milímetros (F15 e F20) e Vigas reforçadas com a forma perdida de dimensões 15 e 20 milíetros (CON+F15 e CON+F20). Nas cinco situações, a peça possui seção transversal de $15 \times 30 \mathrm{~cm}$ e comprimento igual a $2,1 \mathrm{~m}$ e foi considerada situação de flexão de 4 pontos, conforme esquema presente nas figuras 2 e 3 . Para cada situação, calculou-se o máximo deslocamento transversal no vão para um determinado valor de carregamento P. Quanto às propriedades dos materiais, considerou-se os módulos de elasticidade do concreto simples, concreto reforçado com fibra e aço iguais a, respectivamente, $25 \mathrm{MPa}$, 30,05 MPa e $210 \mathrm{MPa}$.

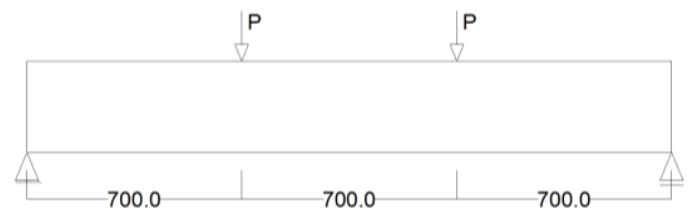

Figura 1: Esquema de apoios e carregamentos da viga sob flexão de 4 pontos.
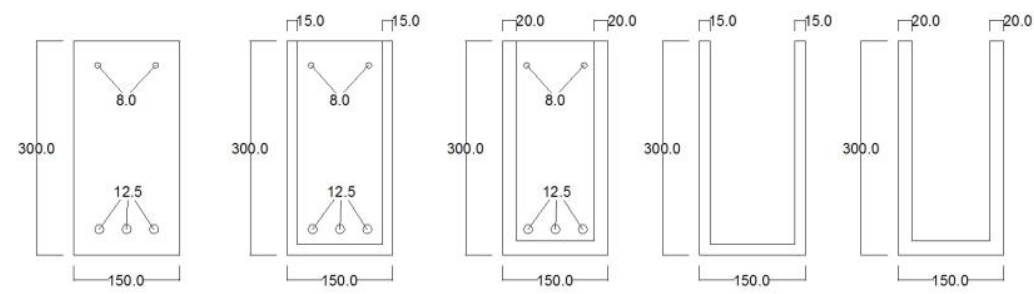

CON

$\mathrm{CON}+\mathrm{F} 15$

$\mathrm{CON}+\mathrm{F} 20$

F15

F20

Figura 2: Seção transversal das vigas testadas. Dimensões em milímetros (mm).

Tabela 1. Deslocamentos na viga de concreto armado sem forma perdida (CON) $\mathrm{P}=11 \mathrm{kN}$.

\begin{tabular}{|c|c|c|c|}
\hline \multicolumn{3}{|c|}{ Deslocamento transversal máximo $(\mathrm{mm})$} & \multirow{2}{*}{ Erro $(\%)$} \\
\hline \multirow{6}{*}{$\begin{array}{l}\text { Resultado } \\
\text { numérico }\end{array}$} & analítico & 0,375069 & \\
\hline & 6 elementos & 0,375056 & $-0,004$ \\
\hline & 12 elementos & 0,37507 & 0,000 \\
\hline & 18 elementos & 0,375073 & 0,001 \\
\hline & 24 elementos & 0,375074 & 0,001 \\
\hline & 30 elementos & 0,375074 & 0,001 \\
\hline
\end{tabular}

Tabela 2. Deslocamentos na forma de espessura 15 milímetros (F15) com $\mathrm{P}=4 \mathrm{kN}$.

\begin{tabular}{l|cccc}
\hline \multicolumn{2}{c|}{ Deslocamento transversal máximo $(\mathrm{mm})$} & \multirow{2}{*}{ Erro $(\%)$} \\
& Resultado analítico & 0,111628 & \\
Resultado & 6 elementos & 0,111485 & $-0,128$ \\
numérico & 12 elementos & 0,111592 & $-0,032$ \\
& 18 elementos & 0,111612 & $-0,014$ \\
& 24 elementos & 0,111618 & $-0,009$ \\
& 30 elementos & 0,111622 & $-0,005$ \\
\hline
\end{tabular}

Tabela 3. Deslocamentos na forma de espessura 20 milímetros (F20) com P=5kN.

\begin{tabular}{c|c|c|c}
\hline \multicolumn{2}{c|}{ Deslocamento transversal máximo $(\mathrm{mm})$} & \multirow{2}{*}{ Erro (\%) } \\
\cline { 1 - 2 } \multicolumn{2}{c}{ Resultado analítico } & 0,086463 & \\
Resultado & 6 elementos & 0,086369 & $-0,109$ \\
\hline
\end{tabular}




\begin{tabular}{c|ccc}
\hline numérico & 12 elementos & 0,086440 & $-0,027$ \\
& 18 elementos & 0,086453 & $-0,012$ \\
& 24 elementos & 0,086457 & $-0,007$ \\
& 30 elementos & 0,086460 & $-0,004$ \\
\hline
\end{tabular}

Tabela 4. Deslocamentos na viga com forma de 15 milímetros (CON+F15) com P=12 kN.

\begin{tabular}{|c|c|c|c|}
\hline \multicolumn{3}{|c|}{ Deslocamento transversal máximo $(\mathrm{mm})$} & \multirow{2}{*}{ Erro $(\%)$} \\
\hline \multirow{6}{*}{$\begin{array}{l}\text { Resultado } \\
\text { numérico }\end{array}$} & analítico & 0,388432 & \\
\hline & 6 elementos & 0,388404 & $-0,007$ \\
\hline & 12 elementos & 0,388425 & $-0,002$ \\
\hline & 18 elementos & 0,388429 & $-0,001$ \\
\hline & 24 elementos & 0,388430 & 0,000 \\
\hline & 30 elementos & 0,388430 & 0,000 \\
\hline
\end{tabular}

Tabela 5. Deslocamentos na viga com forma de 20 milímetros (CON+F20) com $\mathrm{P}=12 \mathrm{kN}$.

\begin{tabular}{|c|c|c|c|}
\hline \multicolumn{3}{|c|}{ Deslocamento transversal máximo $(\mathrm{mm})$} & \multirow{2}{*}{ Erro (\%) } \\
\hline \multirow{6}{*}{$\begin{array}{l}\text { Resultado } \\
\text { numérico }\end{array}$} & analítico & 0,382972 & \\
\hline & 6 elementos & 0,382943 & $-0,008$ \\
\hline & 12 elementos & 0,382965 & $-0,002$ \\
\hline & 18 elementos & 0,382969 & $-0,001$ \\
\hline & 24 elementos & 0,382970 & $-0,001$ \\
\hline & 30 elementos & 0,382971 & 0,000 \\
\hline
\end{tabular}

Foi possível verificar desse conjunto de resultados que, além dos resultados numéricos serem bastante precisos, a convergência ocorreu de forma rápida.

\section{CONSIDERAÇÕES FINAIS}

Os resultados obtidos e a baixa discrepância entre valores analíticos e numéricos evidenciam a eficácia da modelagem, enquanto a rápida convergência evidencia a qualidade do tratamento numérico dado à situação modelada. Para a garantia da robustez da modelagem e tratamento numérico realizados, faz-se necessário a análise não-linear física seguida de ensaios de laboratório a fim de se comparar resultados e convergências para situações mais realísticas.

\section{REFERÊNCIAS}

[1] NAAMAN, A.E. Half a century of progress leading to ultra-high performance diber reinforced concrete: Part 1-overall review. In: 2nd International RILEM Conference on Strain Hardening Cementitious Composites. Rio de Janeiro, 2011. p.17-26.

[2] LIMA, P. R. L., BARROS, J. O., SANTOS, D. J., FONTES, C. M. A., LIMA, J. M. F., TOLEDO FILHO, R. D. Experimental and numerical analysis of short sisal fiber-cement composites produced with recycled matrix. European Journal of Environmental and Civil Engineering, v. 1, p. 1-15, 2017.

[3] NEVES, J. B. Aplicação do Método das Diferenças Finitas Energéticas na Modelagem do Concreto Reforçado com Fibras Curtas de Aço sob Flexão. Feira de Santana; 2012. [Dissertação de Mestrado - PPGECEA, Universidade Estadual de Feira de Santana].

[4] LIMA, J.M.F., NAGAHAMA. K.J., LIMA, P.R.L., SANTOS, G. J. B dos. Um modelo para análise não linear física da flexão de vigas de concreto armado. Mecânica Computacional, vol. XXIX, 2010, p.9839-9853. 